\title{
Étude ethnobotanique des plantes médicinales utilisées dans le traitement du diabète, et des maladies cardiaques dans la région d'Izarène (Nord du Maroc)
}

\author{
Hicham ORCH, Allal DOUIRA et Lahcen ZIDANE \\ Laboratoire de Botanique et de Protection des Plantes, Département de Biologie, Faculté des Sciences, BP. 133, \\ Université lbn Tofaill, Kénitra, Maroc. \\ Correspondant auteur email : zidanelahcenfsk@yahoo.fr
}

Original submitted in on $1^{\text {st }}$ December 2014. Published online at www.m.elewa.org on $28^{\text {th }}$ February 2015

http://dx.doi.org/10.4314/jab.v86i1.3

\begin{abstract}
RÉSUMÉ
Objectif : Dans le but de contribuer à une meilleure connaissance des plantes médicinales utilisées dans le traitement du diabète et de l'hypertension artérielle, auprès de la population riveraine de la forêt d'Izarène. Méthodologie et résultats : Des enquêtes ethnobotaniques ont été réalisées à l'aide de 264 fiches questionnaires dans la région étudiée. Les résultats obtenus ont permis d'inventorier 57 espèces de plantes médicinales appartenant à 30 familles et réparties en 52 genres. Parmi ces espèces, 40 sont traditionnellement utilisées contre le diabète et 30 contre l'hypertension artérielle. Parmi les 40 espèces utilisées dans le traitement du diabète, 13 sont également employées pour traiter l'hypertension artérielle. II s'agit de: Ajuga iva (Ivette musquée), Centaurium Erythraea (Petite centaurée), Origanum compactum (Origan), Rosmarinus officinalis (Romarin), Nigella sativa (Nigelle cultivée), Pistacia lentiscus (Lentisque), Tetraclinis articulata (Thuya), Arbutus unedo (Arbousier), Trigonella foenum-graecum (Fenugrec), Lavandula dentata (Lavande dentée), Salvia officinalis (Calamenthe vulgare), Eugenia caryophyllata (Giroflier), et Olea europaea (Olivier). Les feuilles sont les organes les plus utilisés. La décoction est le mode de préparation dominant et l'administration se fait majoritairement par voie orale. Parmi les 57 espèces médicinales utilisées par la population riveraine, 18 espèces (soit $31.57 \%$ ) spontanées sont collectées de la forêt d'Izarène. La surexploitation menace certaines espèces peu abondantes, comme Origanum compactum, Centaurium Erythraea et Salvia verbenaca (Sauge à feuilles de verveine) de disparition de la forêt d'Izarène. D'où l'intérêt d'adopter une approche de gestion durable pour assurer la conservation et la préservation de ces espèces. Conclusion et application de la recherche : Ce travail constitue une source d'informations pouvant servir de base aux phytochimistes et pharmacologues intéressés par les recherches sur les plantes à effet antidiabétiques et antihypertensives.
\end{abstract}

Mots- clés : Plantes médicinales, forêt d'Izarène, questionnaire, diabète, hypertension artérielle.

\begin{abstract}
Objective: To contribute to a better understanding of medicinal plants used in the treatment of diabetes and high blood pressure, with the riverside population of the forest of Izarène.

Methodology and results : Ethnobotanical surveys were conducted with 264 questionnaires sheets in the study area. The results obtained allowed an inventory of 57 medicinal plant species belonging to 52 genera
\end{abstract}




\section{Orch et al. J. Appl. Biosci. Étude ethnobotanique des plantes médicinales utilisées dans le}

traitement du diabète, et des maladies cardiaques dans la région d'Izarène (Nord du Maroc)

and 30 families. Of these, 40 are traditionally used against diabetes and 30 against hypertension. Of the 40 species used in the treatment of diabetes, 13 are also used to treat high blood pressure. These are: Ajuga iva (Ivette musky), Centaurium erythraea (Centaury), Origanum compactum (Oregano), Rosmarinus officinalis (Rosemary), Nigella sativa (Fennel Flower), Pistacia lentiscus (Mastic), Tetraclinis articulata (Thuja), Arbutus unedo (Arbutus), Trigonella foenum-graecum (Fenugreek), Lavandula dentata (Toothed Lavender), Salvia officinalis (Calamenthe vulgare), Eugenia caryophyllata (Clove) and Olea europaea (The Olive Tree). The leaves are the most used organs. The decoction is the dominant mode of preparation and administration is done mostly orally. Of the 57 medicinal species used by the local population, 18 species $(31.57 \%)$ spontaneous is collected from the forest of Izarène. Overexploitation threatens some scarce species such as, Origanum compactum, Centaurium Erythraea, and Salvia verbenaca (sage verbena leaves) disappearance of the Izarène forest. Hence the need to adopt a sustainable management approach for the conservation and preservation of these species.

Conclusion and application of research: This work is a source of information that can be the basis for phytochemists and pharmacologists interested in research on plants antidiabetic and antihypertensive effect

Keywords : Medicinal plants, Izarene forest, Questionnaire, Diabete, Arterial hypertension.

\section{INTRODUCTION}

À l'instar de plusieurs pays en développement, le Maroc est confronté à l'émergence de maladies chroniques dont le traitement et le suivi constituent, pour lui, un problème économique supplémentaire. Les maladies non transmissibles (MNT) telles que les cardiopathies, le diabète et le cancer sont désormais des problèmes de santé publique. Selon l'OMS, sur les 57 millions de décès survenus dans le monde en 2008, 36 millions $(63 \%)$ étaient dus à des maladies non transmissibles (MNT). La croissance démographique et l'allongement de la durée de vie se traduisent par une augmentation rapide du nombre d'adultes d'âge moyen et avancé, s'accompagnant d'un accroissement correspondant du nombre de décès imputables à une MNT (OMS, 2012). Le diabète est l'une des maladies non transmissibles les plus répandues dans le monde (Jayakumar et al., 2010), il est directement responsable de $3,5 \%$ des décès par MNT (OMS, 2012). Au Maroc, comme ailleurs, il constitue un véritable fléau social dont les conséquences en termes de morbidité et de mortalité sont sévères. Les maladies cardiovasculaires sont à l'origine du plus fort pourcentage de décès par MNT (48\%) dans le monde, et représentent la première cause de décès prématuré de l'adulte (OMS, 2012).
L'hypertension artérielle (HTA) est l'un des principaux déterminants de ces maladies; elle constitue un problème majeur de santé publique aussi bien dans les pays développés que dans les pays en voie de développement. Les régimes alimentaires inadaptés (l'augmentation de la consommation d'alcool, accroissement de la consommation de graisses saturées, réduction de la consommation de fibres) et les modes de vie malsains (l'obésité, la sédentarité, la réduction des activités physiques, le stress de la vie moderne), avec d'autres facteurs de risque ont engendré une prévalence accrue des maladies cardiovasculaires, et du diabète sucré (M.S, 2007). En effet, la prévalence du diabète au Maroc est estimée à 6,6\% de la population âgée de plus de 20 ans et dépasse $10 \%$ après 50 ans (Lefèbvre, 2008). Alors que celle de l'HTA est estimée à $34 \%$ des adultes âgés de plus de 20 ans. Cette prévalence augmente significativement avec l'âge, elle est de $53.8 \%$ chez les personnes âgées de plus de 40 ans et de $72.2 \%$ chez les 65 ans et plus (RBPM, 2012). Face à l'expansion de ces maladies dont la prise en charge est élevée, I'OMS, a encouragé des études ethnobotaniques et des recherches pharmaceutiques pour améliorer des médicaments à base des plantes médicinales afin de promouvoir leurs utilisations optimales 


\section{Orch et al. J. Appl. Biosci. Étude ethnobotanique des plantes médicinales utilisées dans le}

traitement du diabète, et des maladies cardiaques dans la région d'Izarène (Nord du Maroc)

dans les systèmes de prestation des soins de santé (Ghourri et al., 2013; Trabi et al., 2008). Au Maroc, et comme partout dans le monde les plantes continuent de répondre à un besoin important malgré l'influence croissante du système sanitaire moderne. Les médicaments à base de plantes sont considérés comme peu toxiques et doux par rapport aux médicaments pharmaceutiques (Tahri et al., 2012). L'utilisation des plantes pour traiter des maladies chroniques, comme le diabète sucré et l'hypertension artérielle, fait partie d'une tradition marocaine ancienne. Plusieurs auteurs ont mis l'accent sur cette pratique, et les études menées dans différentes régions ont montré que l'usage des plantes pour traiter ces maladies chroniques est très répandu. Les fréquences d'utilisation des plantes, variant de 55 à $90 \%$ des patients, selon les régions (Ziyyat et al., 1997; Jouad et al., 2001; Eddouks et al., 2002; Tahraoui et al., 2007). Les communautés rurales sont considérées comme le secteur le plus négligé

\section{MATERIEL ET METHODES}

Description de la zone d'étude : Selon le découpage administratif en 2010, Le massif forestier d'Izarène fait partie de la région Tanger-Tetouan. II se situe dans la zone Nord-Ouest du Royaume. Au Nord-est de la ville d'Ouezzane et couvre une superficie forestière approximative de 14600 ha. II se trouve au début de la zone mésorifaine externe, entre les parallèles $34^{\circ} 45^{\prime}$ et $34^{\circ} 58^{\prime} \mathrm{N}$ et les méridiens $5^{\circ} 25^{\prime}$ et $5^{\circ} 32^{\prime} \mathrm{W}$, limité à la partie sud par des marnes de la nappe du crétacé pré rifaine avec une altitude variante entre 350 et $680 \mathrm{~m}$ environ (SPEF, 2004). La zone d'étude, le Cercle de Mokrisset, fait partie de la province d'Ouezzane et regroupe 3 Caïdats (Zoumi, Mokrisset et Brikcha), et 3 communes rurales (Zoumi, Ain Baïda et Brikcha) (Figure 1); elle renferme une population estimée à 25 000 habitants (SPEF, 2004).La formation géologique du massif forestier se caractérise par un relief tourmenté du à la présence de plusieurs collines formant le début des montagnes de la chaîne Rifaine du côté sud. Ces collines sont caractérisées par un sol argilo- shisteux ou argilo- marneux profond qui peut atteindre dans des endroits plus de 3 mètres de en termes d'études ethnobotaniques (Prance, 1991), et plusieurs études ont prouvé que jusqu' à $70 \%$ de cette population dépend toujours de la médecine traditionnelle comme source primaire de soins médicaux tandis que seulement $30 \%$ se fondent sur le système conventionnel de soins médicaux (Jeruto, 2008). Cependant, l'exploitation intensive des espèces végétales pour des besoins médicinaux peut devenir néfaste si elle dépasse le seuil tolérable de renouvellement et de régénération des ressources utilisées (Mehdioui \& Kahouadji, 2007). La présente étude est un travail préliminaire que nous avons entrepris, basé sur des enquêtes ethnomédicinales dans l'objectif de recenser les plantes médicinales utilisées dans le traitement du diabète et de l'hypertension artérielle par la population riveraine de la forêt d'Izarène qui présente une diversité floristique, écologique, et offre à la population locale une connaissance assez riche en phytothérapie traditionnelle.

profondeur. Le climat de la zone est subhumide à hiver tempéré, les précipitations moyennes annuelles seraient d'environ $1000 \mathrm{~mm}$. Ces précipitations se répartissent sur environ 70 jours durant toute l'année (Novembre jusqu'au avril). Un hiver doux pratiquement sans gelée et très pluvieux succède une saison sèche et chaude d'une durée de 3 à 4 mois (Askarn, 1982). Le couvert végétal se caractérise par une formation forestière riche et diversifiée, constituée principalement par une végétation qui témoigne d'une dégradation de la formation climacique du chêne liège (Borgniet et al., 2009). Les incendies de forêt constituent pour la région, l'un des principaux facteurs de la dégradation et de destruction. La forêt d'Izarène a une importance économique, écologique et sociale vitale pour la population riveraine. Elle assure les besoins de la population en bois de feu et d'œuvre, et constitue la principale source de fourrage pour le bétail (SPEF, 2004). Au-delà de ses fonctions primordiales, elle joue aussi un rôle dans la médecine traditionnelle grâce à l'utilisation des plantes médicinales qui font partie des moyens de subsistance de la population riveraine. 


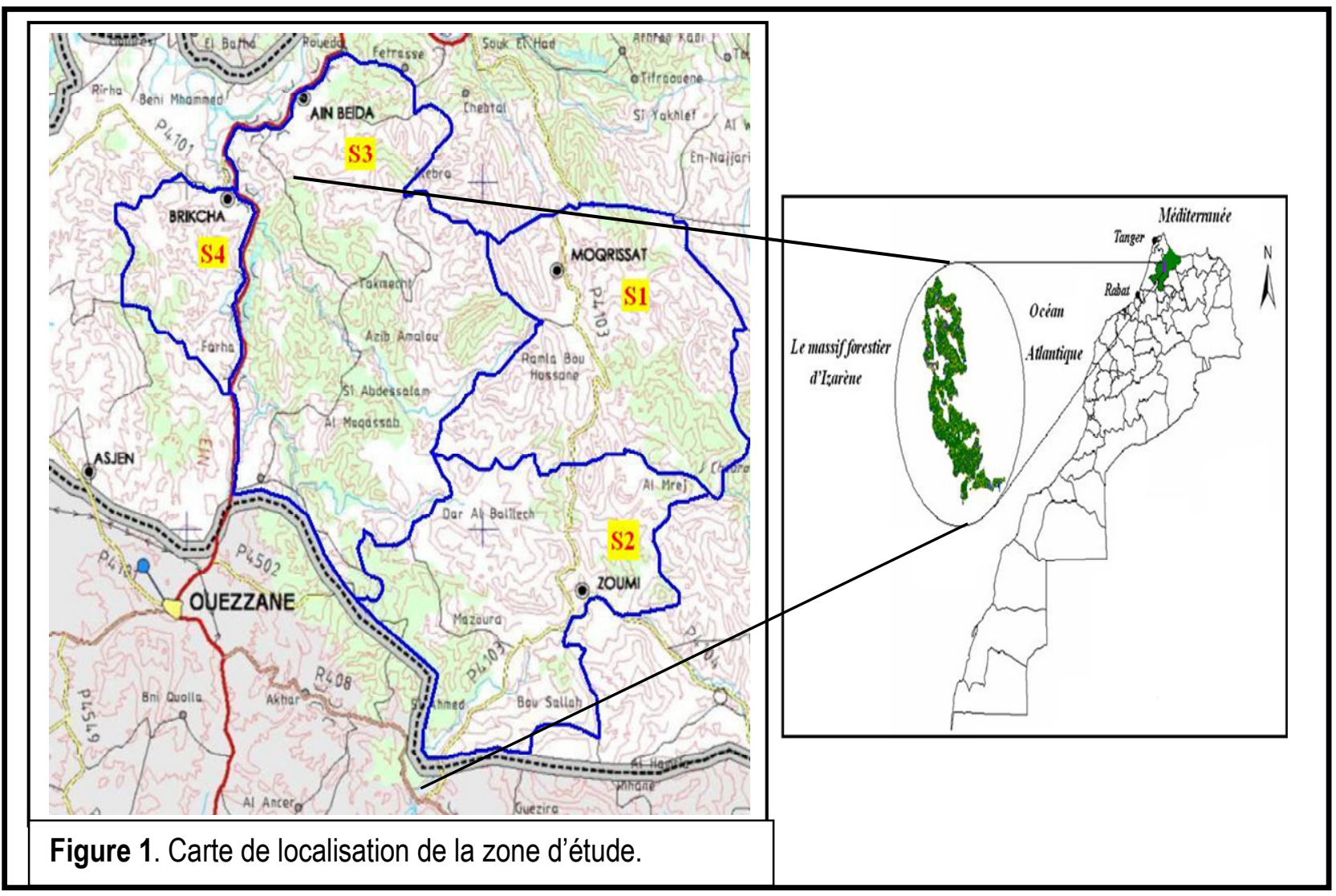

Méthodologie : L'étude prospective a été réalisée entre Février 2007 et Mai 2009 à travers des enquêtes ethnobotaniques dans différentes localités, villages et douars, riveraines de la forêt d'Izarène. La détermination des différents milieux d'enquêtes a été réalisée grâce aux techniques d'échantillonnage stratifié probabiliste (Godron, 1971, 1982). Dans cette étude, l'échantillon est divisé en 4 strates homogènes (S1, S2, S3 et S4), dont 3 correspondent aux nombres des communes rurales du Cercle de Mokrisset (Tableau 1).

Tableau 1. Répartition des enquêtes en fonction des strates.

\begin{tabular}{l|l|l}
\hline Strate & Noms des strates & Nombre d'enquêtes \\
\hline Strate 1 & Mokrisset & 66 \\
\hline Strate 2 & Zoumi & 66 \\
\hline Strate 3 & Ain Baïda & 66 \\
\hline Strate 4 & Brikcha & 66 \\
\hline Échantillon & \multicolumn{2}{|l}{264} \\
\hline
\end{tabular}

L'enquête a été effectuée auprès de 264 personnes habitant villages et douars de la zone étudiée. En adoptant un échantillonnage stratifié aléatoire, des échantillons de 66 personnes sont ensuite formés pour chacune des 4 strates et ils sont mis ensemble pour constituer l'échantillon global (264 personnes). Les études sur le terrain ont porté sur deux genres de personnes : ceux qui ont connu et/ou ont employé des plantes pour des buts médicinaux et ceux qui ont employé des plantes et des produits des plantes pour des buts commerciaux (Collecteurs des plantes, herboristes, tradipraticiens). Un questionnaire préétabli a été conçu pour collecter toute l'information sur l'enquêté et sur l'usage des plantes médicinales par la population locale, notamment le nom vernaculaire de chaque espèce, les méthodes de récolte, les organes 


\section{Orch et al. J. Appl. Biosci. Étude ethnobotanique des plantes médicinales utilisées dans le}

traitement du diabète, et des maladies cardiaques dans la région d'Izarène (Nord du Maroc)

ou la ou les parties de la plante utilisée, le mode de préparation, le mode d'administration, les effets secondaires, etc. Le temps consacré à chaque entrevue était d'environ deux heures et toutes les personnes interrogées ont été informées sur l'objectif de cette étude. La liste floristique complète était établie après détermination et vérification des échantillons récoltés sur le terrain, grâce aux documents suivants :

Petite flore des régions arides du Maroc occidental de Négre $(1961,1962)$, tomes I et II.

Nouvelle flore d'Algérie et des régions désertiques méridionales de Quézel \& Santana $(1962,1963)$, tomes I et II.

\section{RESULTATS ET DISCUSSION}

Les enquêtes ethnobotaniques réalisées sur le terrain ont permis d'interroger 264 personnes dont $74,36 \%$ de sexe féminin contre $25,64 \%$ de sexe masculin. De même, les résultats obtenus montrent que ce sont les femmes qui utilisent beaucoup plus les plantes médicinales que les hommes. Ces résultats confirment les résultats d'autres travaux ethnobotaniques réalisés à l'échelle nationale (Ziyyat et al., 1997; Hmamouchi, 2001; Jouad et al., 2001; Eddouks et al., 2002; Tahraoui et al., 2007; Mehdioui \& Kahouadji, 2007; Salhi et al., 2010; Benkhnigue et al., 2010 ). L'âge variait de 25 à 84 ans, et les sujets d'au moins soixante (60) ans représentent plus du tiers de la population étudiée. En effet, les personnes âgées sont sensées fournir des informations plus fiables, du fait qu'elles détiennent une bonne partie du savoir ancestral qui se transmet oralement. La transmission de cette connaissance est en danger actuellement parce qu'elle n'est pas toujours assurée (Weniger, 1991 ; Anyinam 1995). $75 \%$ des personnes enquêtées étaient analphabètes ou avaient un niveau scolaire primaire.

L'étude des plantes médicinales a permis de recenser et d'identifier 57 espèces appartenant à 30 familles et réparties en 52 genres, avec 40 étant employés pour le diabète, 30 pour l'hypertension, et 13 pour les deux maladies. Les familles les plus représentées sont les Lamiaceae (12 espèces), les Apiaceae (5 espèces) et les Asteraceae (4 espèces), viennent ensuite les Myrtaceae, Rosaceae et Solanaceae (3 espèces). Les autres familles restantes ne comptent qu'une ou deux espèces (27 espèces). Cette représentativité a également été observée, à quelques différences près, au cours des enquêtes ethnomédicinales réalisées dans d'autres régions du pays par Jouad et al. (2001), par Eddouks et al. (2002), et par Tahraoui et al. (2007).
Les plantes médicinales du Maroc de Sijelmassi (1993).

La pharmacopée marocaine traditionnelle de Bellakhder (1997).

Flore pratique du Maroc de Fennane et al. (1999).

Les plantes médicinales et aromatiques marocaines de Hmamouchi (2001), 2ème édition.

Catalogues des plantes vasculaires du Nord du Maroc, incluant des clés d'identification, de Valdés et al. (2002), volumes I et II.

Flore vasculaire du Maroc : inventaire et chorologie de Fennane \& Ibn Tattou (2005).

Parmi les 57 espèces médicinales utilisées par la population riveraine, 23 espèces (soit 40.35\%) sont importées d'autres régions du pays, 16 espèces (soit $28.07 \%$ ) sont cultivées, alors que 18 espèces (soit $31.57 \%$ ) spontanées sont collectées de la forêt d'Izarène. Parmi ces dernières, trois plantes médicinales, Origanum compactum, Centaurium Erythraea et Salvia verbenaca sont peu abondantes dans la région et risquent de disparaitre de la forêt d'Izarène si aucune mesure de protection n'est prise par les services concernés, en raison de la collecte intensive de ces espèces. Dans notre étude, le dépouillement des données collectées sur le terrain nous a permis de déterminer quarante (40) plantes différentes antidiabétiques (Tableau 2). Les plus fréquemment utilisées par les sujets diabétiques étaient: Trigonella foenum-graecum (Fenugrec), Artemisia herba-alba (Armoise blanche), Ammi visnaga (Ammi visnage), Centaurium erythreae (Petite centaurée), Myrtus communis (Myrte), Globularia alypum (Globulaire turbith), Nigella sativa (Nigelle cultivée), Zygophyllum gaetulum (Zygophylle), Olea europaea (Olivier), Rosmarinus officinalis (Romarin), Marrubium vulgare (Marrube blanc), Allium cepa (Oignon), Ajuga iva (Ivette musquée), Salvia officinalis (Calamenthe vulgare), Artemesia absinthium(Absinth), Prunus amygdalus (Amandier), Capsicum frutescens (Piment enragé), Origanum compactum (Origan), Nerium oleander (Laurier rose), et Urtica dioica (Ortie). Certaines de ces plantes ont été signalées par des enquêtes ethnomédicinales récentes dans le traitement du diabète (Jouad et al., 2001 ; Eddouks et al., 2002 ; Tahraoui et al., 2007 ; Ghourri et al., 2013). Parmi ces plantes figurent Zygophyllum gaetulum, Olea europaea, Marrubium vulgare, Trigonella foeniculum-graecum, 


\section{Orch et al. J. Appl. Biosci. Étude ethnobotanique des plantes médicinales utilisées dans le}

traitement du diabète, et des maladies cardiaques dans la région d'Izarène (Nord du Maroc)

Nerium oleander, Rosmarinus officinalis et Nigella sativa. L'activité antidiabétique de certaines plantes a également été prouvée expérimentalement par des études in vivo ou in vitro (Tableau 2), à titre d'exemple l'effet antihyperglycémique de l'armoise blanche (Artemisia herba-alba) (Twaij et al-Badr, 1988; AlKhazraji et al., 1993; Marrif et al., 1995; Tastekin et al., 2006; Mansi et al., 2007), de l'ammi visnage (Ammi visnaga) (Jouad et al., 2002), de l'ortie (Urtica dioica) (Bnouham et al., 2003), de l'oignon (Allium cepa) (Kelkar et al., 2001; Ozougwu, 2011), du marrube blanc (Marrubium vulgare) (Roman- Ramos et al., 1992; Herrera-Arellano et al., 2004), du nigelle cultivée (Nigella sativa) (Al-Hader et al., 1993), du zygophylle (Zygophyllum gaetulum) (Jaouhari et al., 1999; Skim et al., 1999), de l'olivier (Olea europaea) (Circosta et al., 1986; Al Jamal et Ibrahim, 2011), et du fenugrec (Trigonella foenum-graecum) (Abdel-Barry et al., 1997; Alarcon-Aguilara et al., 1998; Gupta et al., 2001 ; Eidi et al., 2007). Notons que le fenugrec a également fait l'objet d'études cliniques (Sharma et al., 1990). L'effet hypoglycémique du romarin (Rosmarinus officinalis) avait également été démontré chez des souris normales et rendues diabétiques (Erenmemisoglu et al., 1997). Cependant, la petite centaurée (Centaurium erythreae) n'a été citée, pour son activité antidiabétique, que dans trois enquêtes (Jouad et al., 2001; Bnouham et al., 2002; Eddouks et al., 2007).

Trente (30) plantes sont antihypertensives (Tableau 2). Les plus sollicitées sont: Allium sativum(Ail), Olea europaea (Olivier), Rosmarinus officinalis (Romarin), Nigella sativa (Nigelle cultivée), Petroselinum sativum (Persil), Ajuga iva (Ivette musquée), Tetraclinis articulate (Thuya), Thymus vulgaris (Thym), Inula viscosa (Inule visqueuse), Eugenia caryophyllata (Giroflier), Mentha pulegium (Menthe pouliot), Salvia verbenaca (Sauge verveine), et Arbutus unedo (Arbousier). Comme dans le cas du diabète, certaines des plantes ont été signalées par des enquêtes ethnomédicinales récentes dans le traitement de l'hypertension artérielle et les problèmes cardiovasculaires. Parmi ces plantes figurent Petroselinum sativum, Allium sativum, Tetraclinis articulate, Rosmarinus officinalis, Thymus vulgaris, Eugenia caryophyllata, et Olea europaea (Jouad et al., 2001 ; Eddouks et al., 2002 ; Tahraoui et al., 2007 ). L'activité antihypertensive de quelques plantes a également été prouvée expérimentalement. C'est le cas de l'ail (Allium sativum) (Malik et Siddiqui, 1981; Pantoja et al., 1991,
1996 ; Andrianova et al., 2002), de l'arbousier (Arbutus unedo) (Fitzpatrick et al., 1993; Abdalla et al., 1994), du Romarin (Rosmarinus officinalis) (Aqel, 1991), de la nigelle cultivée (Nigella sativa) (Labhal et al., 1994) et de l'olivier (Olea europaea) (Circosta et al., 1986) (Tableau 2). Ces résultats qui confirment l'activité biologique de ces plantes expliquent, en partie, leur utilisation dans le traitement du diabète et de l'hypertension artérielle par la population riveraine de la forêt d'Izarène. Parmi les 40 espèces utilisées dans le traitement du diabète, treize (13) sont utilisées pour traiter aussi bien le diabète que l'hypertension artérielle et les maladies cardiaques. II s'agit de: Ajuga iva, Centaurium Erythraea, Origanum compactum, Rosmarinus officinalis, Nigella sativa, Pistacia lentiscus, Tetraclinis articulate, Arbutus unedo, Trigonella foenum-graecum, Lavandula dentata, Salvia officinalis, Eugenia caryophyllata, et Olea europaea. Ce résultat montre bien le lien étroit entre l'hypertension artérielle et le diabète, en effet, certaines plantes que nous avons notées ouvrent des perspectives intéressantes dans la recherche des nouveaux moyens thérapeutiques, pouvant ainsi apporter des solutions crédibles par la réalisation de médicaments à faibles coûts et efficaces pour le traitement simultané de l'hypertension artérielle et du diabète. Par ailleurs, Parmi les plantes antidiabétiques et antihypertensives recensées, certaines sont reconnues par leur pouvoir toxique tels : Nerium oleander (Langford et Boor, 1996 ; Al-Yahya et al., 2000), Nigella sativa (Zaoui et al., 2002; Ali et Blunden, 2003), Zygophyllum gaetulum et Artemisia herba-alba (Eddouks et al., 2002 ; Tahraoui et al., 2007). En effet, la majorité des phytothérapeutes ignorent la toxicité des plantes utilisées ainsi que les modalités de leur usage, notamment les modes de préparation et les doses préconisées. L'utilisation des plantes médicinales doit être rationalisée pour en tirer profit et éviter les risques. Des études portant sur ces objectifs sont donc nécessaires. Par ailleurs, les résultats obtenus montrent également que les feuilles sont les organes les plus utilisés, suivies par les fruits, les racines et les écorces. De même, la décoction et l'administration sous forme de poudre, sont les principaux modes de préparation et d'administration. Cette prescription peut s'expliquer par le fait que les deux maladies sont liées à des organes profonds. Pour les atteindre, tout composé doit transiter par l'appareil digestif pour en faciliter son assimilation (Trabi et al., 2008). 
Tableau 2 : Liste des plantes recensées dans la région d'étude et les pathologies qui leur sont associées, et les études expérimentales qui prouvent leur utilisation. ( $\mathrm{D}$ : diabètes; $\mathrm{H}$ : hypertension)

\begin{tabular}{|c|c|c|c|c|c|c|}
\hline \multirow[t]{2}{*}{ Nom de la plantes } & \multirow[t]{2}{*}{ Nom commun } & \multicolumn{2}{|c|}{ Pathologies } & \multirow{2}{*}{$\begin{array}{l}\text { Partie de la } \\
\text { plante utilisée }\end{array}$} & \multirow{2}{*}{$\begin{array}{l}\text { forme } \\
\text { administrées }\end{array}$} & \multirow[t]{2}{*}{ Références } \\
\hline & & D & $H$ & & & \\
\hline $\begin{array}{l}\text { Amaryllidaceae } \\
\text { Allium sativum } \mathrm{L} \text {. } \\
\text { Allium cepa L. }\end{array}$ & $\begin{array}{l}\text { Thouma } \\
\text { Al'Bassla }\end{array}$ & $x$ & $x$ & $\begin{array}{l}\text { Bulbe } \\
\text { Bulbe }\end{array}$ & $\begin{array}{l}\text { Cru } \\
\text { Cru }\end{array}$ & $\begin{array}{l}\text { Malik et Siddiqui (1981) } \\
\text { Sial et Ahmad (1982) } \\
\text { Pantoja et al. (1991) (1996) } \\
\text { Andrianova et al. (2002) } \\
\text { Mathew et Augusti (1975) } \\
\text { Roman-Ramos et al. (1995) } \\
\text { Sheela et al. (1995) } \\
\text { Kelkar et al. (2001) } \\
\text { Kumari et Augusti (2002) } \\
\text { Campos et al. (2003) } \\
\text { El-Demerdash et al. (2005) } \\
\text { Jelodar et al. (2005) } \\
\text { Ozougwu (2011) }\end{array}$ \\
\hline $\begin{array}{l}\text { Anacardiaceae } \\
\text { Pistacia lentiscus } L .\end{array}$ & Adru & $x$ & $x$ & Feuilles & Déco., infu. & \\
\hline $\begin{array}{l}\text { Apiaceae } \\
\text { Ammi visnaga (Lam) } \\
\text { Ammodaucus leucotrichus Coss. } \\
\text { et Dur } \\
\text { Cuminum cyminum L. } \\
\text { Foeniculum vulgare Gaertn. } \\
\text { Petroselinum sativum Hoffm. }\end{array}$ & $\begin{array}{l}\text { Bachnikha } \\
\text { Kamoun sooufi } \\
\quad \text { Kamoun } \\
\text { Nafaâ } \\
\text { Maâdanous }\end{array}$ & $x$ & $\begin{array}{l}x \\
x \\
x \\
x\end{array}$ & $\begin{array}{l}\text { Fruits } \\
\text { Graines } \\
\text { Graines } \\
\text { Graines } \\
\text { Graines, Partie } \\
\text { aérienne } \\
\end{array}$ & $\begin{array}{l}\text { Décoction } \\
\text { Poudre, déco., } \\
\text { infu. } \\
\text { Poudre, déco. } \\
\text { Poudre, déco. } \\
\text { Déco., Cru }\end{array}$ & $\begin{array}{l}\text { Jouad et al. (2002) } \\
\text { Alaoui et al. (1992) } \\
\text { Beaux et al. (1997) } \\
\text { El Bardai et al. (2001) }\end{array}$ \\
\hline $\begin{array}{l}\text { Apocynaceae } \\
\text { Nerium oleander } L \text {. }\end{array}$ & Defla & $x$ & & Feuilles & Déco., infu. & \\
\hline
\end{tabular}


Orch et al. J. Appl. Biosci. Étude ethnobotanique des plantes médicinales utilisées dans le traitement du diabète, et des maladies cardiaques dans la région d'Izarène (Nord du Maroc)

\begin{tabular}{|c|c|c|c|c|c|c|}
\hline $\begin{array}{l}\text { Asteraceae (Composées) } \\
\text { Artemisia absinthium L. } \\
\text { Artemisia herba alba Assac. } \\
\text { Inula viscosa L. Ait. } \\
\text { Matricaria chamomilla L. }\end{array}$ & $\begin{array}{l}\text { Chiba } \\
\text { Chih } \\
\text { Terrahla } \\
\text { Mansania }\end{array}$ & $\begin{array}{l}x \\
x\end{array}$ & $x$ & $\begin{array}{l}\text { Partie aérienne } \\
\text { Feuilles, Partie } \\
\text { aérienne } \\
\text { Feuilles } \\
\text { Feuilles, Fleurs }\end{array}$ & $\begin{array}{l}\text { Déco., infu. } \\
\text { Poudre, déco., } \\
\text { infu. } \\
\text { Décoction } \\
\text { Déco., infu. }\end{array}$ & $\begin{array}{l}\text { Twaij et al-Badr (1988) } \\
\text { Al-Alami et Farjou (1990) } \\
\text { Al-Khazraji et al. (1993) } \\
\text { Al-Shamaony et al. (1994) } \\
\text { Marrif et al. (1995) } \\
\text { Tastekin et al. (2006) } \\
\text { Mansi et al. (2007) }\end{array}$ \\
\hline $\begin{array}{l}\text { Boraginaceae } \\
\text { Borago officinalis } L \text {. }\end{array}$ & Harricha & & $x$ & Feuilles, Fleurs & Déco., infu. & \\
\hline $\begin{array}{l}\text { Brassicaceae (Cruciferae) } \\
\text { Lepidium sativum L. }\end{array}$ & Hab Rchad & & $x$ & Graines & Décoction & Maghrani et al. (2005) \\
\hline $\begin{array}{l}\text { Capparaceae } \\
\text { Capparis spinosa } L \text {. }\end{array}$ & Al'Kabbar & $x$ & & $\begin{array}{l}\text { Partie aérienne, } \\
\text { Fruits }\end{array}$ & Décoction & Eddouks et al. (2004) \\
\hline $\begin{array}{l}\text { Chenopodiaceae } \\
\text { Chenopodium ambrosioides } L \text {. }\end{array}$ & Mkhinza & $x$ & & Feuilles, Fleurs & Déco., infu. & \\
\hline $\begin{array}{l}\text { Cucurbitaceae } \\
\text { Cucurbita pepo } L .\end{array}$ & L'krâa & $x$ & & Graines & Déco., poudre & \\
\hline $\begin{array}{l}\text { Cupressaceae } \\
\text { Tetraclinis articulata Benth. }\end{array}$ & Al'Araâr & $x$ & $x$ & Feuilles & $\begin{array}{l}\text { Poudre, } \\
\text { macération }\end{array}$ & \\
\hline $\begin{array}{l}\text { Ericaceae } \\
\text { Arbutus unedo } \mathrm{L} .\end{array}$ & Sasnou & $x$ & $x$ & Feuilles, racines & Décoction & $\begin{array}{c}\text { Fitzpatrick et al. (1993) } \\
\text { Abdalla et al. (1994) }\end{array}$ \\
\hline $\begin{array}{l}\text { Fabaceae } \\
\text { Trigonella foenum-graecum } \mathrm{L} .\end{array}$ & Al'Houlba & $x$ & $x$ & Graines & $\begin{array}{l}\text { Déco., poudre, et } \\
\text { macération }\end{array}$ & $\begin{array}{l}\text { Riyad et al. (1988) } \\
\text { Swanston-Flatt et al. (1989) } \\
\text { Sharma et al. (1990) } \\
\text { Abdel-Barry et al. (1997) } \\
\text { Alarcon-Aguilar et al. (1998) } \\
\text { Raju et al. (2001) } \\
\text { Gupta et al. (2001) } \\
\text { Devi et al. (2003) } \\
\text { Jelodar et al. (2005) } \\
\text { Eidi et al. (2007) }\end{array}$ \\
\hline
\end{tabular}


Orch et al. J. Appl. Biosci. Étude ethnobotanique des plantes médicinales utilisées dans le traitement du diabète, et des maladies cardiaques dans la région d'Izarène (Nord du Maroc)

\begin{tabular}{|c|c|c|c|c|c|c|}
\hline $\begin{array}{l}\text { Fagaceae } \\
\text { Quercus suber } L .\end{array}$ & D'bbagh & & $x$ & Ecorces & Poudre & \\
\hline $\begin{array}{l}\text { Gentianaceae } \\
\text { Centaurium Erythraea Rafn }\end{array}$ & $\begin{array}{l}\text { Gossat } \\
\text { Al'Hayya }\end{array}$ & $x$ & $x$ & Partie aérienne & Déco., infu. & $\begin{array}{c}\text { Fluck, (1973) } \\
\text { Alaoui et al. (1992) } \\
\text { Hamza et al. (2010) } \\
\text { Haloui et al. (2000) }\end{array}$ \\
\hline $\begin{array}{l}\text { Globulariaceae } \\
\text { Globularia alypum L. }\end{array}$ & Aïn Larneb & $x$ & & Feuilles & Déco., poudre & \\
\hline $\begin{array}{l}\text { Lamiaceae } \\
\text { Ajuga iva (L.) Schreb. } \\
\text { Calamintha officinalis Moench } \\
\text { Marrubium vulgare } L \text {. } \\
\text { Mentha pulegium } L \text {. } \\
\text { Ocimum basilicum } L \text {. } \\
\text { Origanum compactum Bentham. } \\
\text { Lavandula dentata } L \text {. } \\
\text { Lavandula stoechas } L \text {. } \\
\text { Rosmarinus officinalis } L \text {. } \\
\text { Salvia officinalis } L . \\
\text { Salvia verbenaca } L . \\
\text { Thymus ssp (vulgaris) }\end{array}$ & \begin{tabular}{l} 
Chendgora \\
Manta \\
Merriwta \\
\multicolumn{1}{c}{ Fliyou } \\
Lahbak \\
Zaâtar \\
Lakhzama \\
Al'Halhal \\
Azir \\
Assalmiya \\
Al'khiyata \\
Zaîtra
\end{tabular} & $\begin{array}{l}X \\
X \\
X\end{array}$ & $\begin{array}{l}x \\
x \\
x^{x} \\
x \\
x^{x} \\
x\end{array}$ & $\begin{array}{l}\text { Partie aérienne } \\
\text { Partie aérienne } \\
\text { Partie aérienne } \\
\text { Partie aérienne } \\
\text { Partie aérienne } \\
\text { Feuilles } \\
\text { Partie aérienne } \\
\text { Feuilles } \\
\text { Feuilles } \\
\\
\text { Feuilles } \\
\text { Partie aérienne } \\
\text { Partie aérienne }\end{array}$ & $\begin{array}{l}\text { Décoction } \\
\text { Déco., infu. } \\
\text { Décoction } \\
\text { Déco., infu. } \\
\text { Déco., infu. } \\
\text { Infusion } \\
\text { Déco., infu. } \\
\text { poudre } \\
\text { Déco., infu. } \\
\text { Décoction } \\
\text { Infusion } \\
\text { Déco., infu. } \\
\text { Décoction }\end{array}$ & $\begin{array}{l}\text { Aqel, (1991) } \\
\text { Al-Hader et al. (1994) } \\
\text { Erenmemisoglu et al. (1997) } \\
\text { Haloui et al. (2000) } \\
\\
\text { Swanston-Flatt et al. (1989) } \\
\text { Alarcon-Aguilar et al. (2002) } \\
\text { Eidi et al. (2005) }\end{array}$ \\
\hline $\begin{array}{l}\text { Myrtaceae } \\
\text { Eucalyptus globulus Labill (sp.) } \\
\text { Eugenia caryophyllata } \\
\text { Myrtus communis L. }\end{array}$ & $\begin{array}{l}\text { Al' Kalitouss } \\
\text { Qronfel } \\
\text { Arraihan }\end{array}$ & $x$ & $x$ & $\begin{array}{l}\text { Feuilles, Fruits } \\
\text { Feuilles, Clous } \\
\text { Feuilles, Fruits }\end{array}$ & $\begin{array}{l}\text { Décoction } \\
\text { Déco., poudre, et } \\
\text { macération } \\
\text { Déco., infu. }\end{array}$ & $\begin{array}{l}\text { Swanston-Flatt et al. (1990) } \\
\text { Gallagher et al. (2003) } \\
\text { Jouad et al. (2003) } \\
\text { Elfellah et al. (1984) } \\
\text { Sepici et al. (2004) } \\
\text { Onal et al. (2005) }\end{array}$ \\
\hline
\end{tabular}


Orch et al. J. Appl. Biosci. Étude ethnobotanique des plantes médicinales utilisées dans le traitement du diabète, et des maladies cardiaques dans la région d'Izarène (Nord du Maroc)

\begin{tabular}{|c|c|c|c|c|c|c|}
\hline $\begin{array}{l}\text { Oleaceae } \\
\text { Olea europaea var. sativa }\end{array}$ & $\begin{array}{l}\text { Zitoun, } \\
\text { Zabbouj }\end{array}$ & $x$ & $x$ & Feuilles, Fruits & Déco., infu., huile & $\begin{array}{l}\text { Circosta et al. (1986) } \\
\text { Bennani-Kabchi et al. (2000) } \\
\text { Somova, et al. (2003) } \\
\text { Al Jamal et Ibrahim (2011) }\end{array}$ \\
\hline $\begin{array}{l}\text { Pedaliaceae } \\
\text { Sesamum indicum Dc. }\end{array}$ & Ajenjlane & $\mathrm{x}$ & & Graines & Infusion, poudre & \\
\hline $\begin{array}{l}\text { Poaceae } \\
\text { Phalaris canariensis } \\
\text { Zea mays L. }\end{array}$ & $\begin{array}{l}\text { Zwân } \\
\text { Lahyat Adra }\end{array}$ & $\begin{array}{l}x \\
x\end{array}$ & & $\begin{array}{l}\text { Fruits } \\
\text { Stigmates des } \\
\text { fleurs }\end{array}$ & $\begin{array}{l}\text { Déco., infu., } \\
\text { poudre } \\
\text { Déco., infu. }\end{array}$ & Miura et al. (1996) \\
\hline $\begin{array}{l}\text { Ranunculaceae } \\
\text { Nigella sativa L. }\end{array}$ & Assanouj & $x$ & $x$ & Graines & $\begin{array}{l}\text { Décoction, } \\
\text { poudre }\end{array}$ & $\begin{array}{l}\text { Al-Hader et al. (1993) } \\
\text { El Tahir et al. (1993) } \\
\text { Labhal et al. (1994) } \\
\text { Labhal et al. (1999) } \\
\text { Zaoui et al. (2000) } \\
\text { Hawsawi et al. (2001) } \\
\text { El-Dakhakhny et al. (2002) } \\
\text { Kanter et al. (2003) } \\
\text { Fararh et al. (2004) } \\
\text { Le et al. (2004) } \\
\text { Rchid et al. (2004) } \\
\text { Mansi (2006) }\end{array}$ \\
\hline $\begin{array}{l}\text { Rosaceae } \\
\text { Mallus communis } \\
\text { Prunus amygdalus stokes var. } \\
\text { amara D.C. } \\
\text { Rosa fructicosus } L \text {. }\end{array}$ & $\begin{array}{l}\text { Teffah } \\
\text { Louz Morr } \\
\text { Toute chaouki }\end{array}$ & $\begin{array}{l}x \\
x\end{array}$ & $x$ & $\begin{array}{l}\text { Fruits } \\
\text { Graines } \\
\text { Feuilles, Fruits }\end{array}$ & $\begin{array}{l}\text { Cru } \\
\text { Déco., infu. } \\
\text { Déco., infu. }\end{array}$ & \\
\hline $\begin{array}{l}\text { Rubiaceae } \\
\text { Rubia tinctorium L. }\end{array}$ & Al'Fouwa & & $x$ & Racines & Décoction & \\
\hline $\begin{array}{l}\text { Rutaceae } \\
\text { Citrus bigaradia Riss. } \\
\text { Ruta montana L. }\end{array}$ & $\begin{array}{l}\text { Larenj } \\
\text { Al'Fijel }\end{array}$ & $\begin{array}{l}x \\
x\end{array}$ & & $\begin{array}{l}\text { Feuilles, Fleurs } \\
\text { Partie aérienne }\end{array}$ & $\begin{array}{l}\text { Déco., infu. } \\
\text { Déco., infu., } \\
\text { poudre }\end{array}$ & \\
\hline
\end{tabular}


Orch et al. J. Appl. Biosci. Étude ethnobotanique des plantes médicinales utilisées dans le traitement du diabète, et des maladies cardiaques dans la région d'Izarène (Nord du Maroc)

\begin{tabular}{|c|c|c|c|c|c|c|}
\hline $\begin{array}{l}\text { Solanaceae } \\
\text { Mandragora autumnalis Bertol. } \\
\text { Nicotiana tabacum } L \text {. } \\
\text { Capsicum frutescens } L \text {. }\end{array}$ & $\begin{array}{l}\text { Baydh } \\
\text { Al'Ghoul } \\
\text { Nefha } \\
\text { Felfel Hârr }\end{array}$ & $\begin{array}{l}X \\
X\end{array}$ & $x$ & $\begin{array}{l}\text { Racines } \\
\text { Feuilles } \\
\text { Graines }\end{array}$ & $\begin{array}{l}\text { Déco., poudre } \\
\text { Décoction } \\
\text { Cru }\end{array}$ & \\
\hline $\begin{array}{l}\text { Urticaceae } \\
\text { Urtica dioîca } L .\end{array}$ & Hourrika & $x$ & & Partie aérienne & Infusion & $\begin{array}{l}\text { Swanston-Flatt et al. (1989) } \\
\text { Roman Ramos et al. (1992) } \\
\text { Bnouham et al. (2003) } \\
\text { Farzami et al. (2003) } \\
\text { Onal et al. (2005) }\end{array}$ \\
\hline $\begin{array}{l}\text { Verbenaceae } \\
\text { Lippia citriodora }\end{array}$ & Alwiza & $X$ & & Feuilles & Déco., infu. & \\
\hline $\begin{array}{l}\text { Zingiberaceae } \\
\text { Zingiber officinal Rosc. }\end{array}$ & Sekinjbir & $x$ & & Rhizome & $\begin{array}{l}\text { Déco., poudre, et } \\
\text { macération }\end{array}$ & \\
\hline $\begin{array}{l}\text { Zygophyllaceae } \\
\text { Zygophyllum gaetulum Emb. \& } \\
\text { Maire }\end{array}$ & Al'âggaya & $x$ & & Feuilles & $\begin{array}{l}\text { Décoction, } \\
\text { infusion }\end{array}$ & $\begin{array}{l}\text { Skim et al. (1999) } \\
\text { Jaouhari et al., } 1999 \\
\text { Jaouhari et al. (2000) }\end{array}$ \\
\hline
\end{tabular}




\section{Orch et al. J. Appl. Biosci. Étude ethnobotanique des plantes médicinales utilisées dans le}

traitement du diabète, et des maladies cardiaques dans la région d'Izarène (Nord du Maroc)

\section{CONCLUSION}

Le coût onéreux des traitements proposés par la médecine moderne, en plus d'effets secondaires gênants, constitue les principales limites rencontrées lors du traitement. Ces limites peuvent justifier la recherche de nouvelles approches thérapeutiques par l'utilisation de plantes médicinales de la pharmacopée traditionnelle, Ainsi, les plantes médicinales antidiabétiques et antihypertensives inventoriées dans notre étude, peuvent offrir une large réponse au problème complexe du diabète sucré et de l'hypertension artérielle, et des perspectives thérapeutiques pour une meilleure prise en charge. En outre, les enquêtes ethnobotaniques que nous avons menées auprès de la population riveraine de la forêt d'lzarène ont permis de recenser et d'identifier 57 espèces de plantes appartenant à 30 familles et réparties en 52 genres. Quarante (40) de ces plantes sont utilisées contre le diabète, trente (30) contre

\section{RÉFÉRENCES}

Abdalla, S., Abu-Zarga, M., Sabri, M., 1994. Effects of the flavones luteolin, isolated from Colchicum richii on guinea-pig isolated smooth muscle and heart and on blood pressure and blood flow. Phythoterapy Research 8, 265-270.

Abdel-Barry J.A., Abdel-Hassan I.A., Al-Hakiem M.H., 1997. Hypoglycaemic and antihyperglycaemic effects of Trigonella foenum-graecum leaf in normal and alloxan induced diabetic rats. J. Ethnopharmacol.; 58: 149-155.

Al-Alami, A.H.H., Farjou, I.B., 1990. Effect of feeding of Artemisia herba-alba Asso on glucokinase and ATPase activity in normal and diabetic rabbits. Journal of the Faculty of Medicine (Baghdad) 32, 13-25.

Alaoui, T., Benabdelkrim, I., Zaid, A., 1992. Etude de l'effet hypoglycémiant sur des rats d'une association de Ammi visnaga, Centaurium erythraea et Thymus ciliatus utilisées enmédecine traditionnelle Marocaine. Al Biruniya. Revue Marocaine de Pharmacognosie 8, 37-44.

Alarcon-Aguilar, F.J., Roman-Ramos, R., PerezGutierrez, S., Aguilar- Contreras, A., Contreras-Weber, C.C., Flores-Saenz, J.L., 1998. Study of the anti-hyperglycemic effect of plants used as antidiabetics. Journal of Ethnopharmacology 61, 101-110. l'hypertension artérielle, et 13 plantes sont utilisées pour traiter aussi bien l'hypertension artérielle que le diabète. II s'agit de : Ajuga iva, Centaurium Erythraea, Origanum compactum, Rosmarinus officinalis, Nigella sativa, Pistacia lentiscus, Tetraclinis articulate, Arbutus unedo, Trigonella foenum-graecum, Lavandula dentata, Salvia officinalis, Eugenia caryophyllata, et Olea europaea. Le feuillage est la partie la plus utilisée de la plante alors que la décoction est le mode d'emploi le plus pratiqué dans le traitement phytothérapeutique. Ce travail, bien que limité par le nombre de travaux ethnopharmacologiques disponibles consultés, constituent la base d'études ultérieures pour la valorisation des plantes médicinales utilisées contre le diabète et l'hypertension artérielle à travers des études biologiques et photochimiques des plantes inventoriées.

Alarcon-Aguilar, F.J., Roman-Ramos, R., Flores-Saenz, J.L., Aguirre-Garcia, F., 2002. Investigation on the hypoglycaemic effects of extracts of four Mexican medicinal plants in normal and alloxan-diabetic mice. Phytotherapy Research 16, 383-386.

Al-Hader, A.A., Aqel, M., Hasan, Z., 1993. Hypoglycemic effects of the volatile oil of Nigella sativa seeds. International Journal of Pharmacognosy 31, 96-100.

Al-Hader, A.A., Hasan, Z.A., Aqel, M.B., 1994. Hyperglycemic and insulin release inhibitory effects of Rosmarinus officinalis. Journal of Ethnopharmacology 43, 217-221.

Al Jamal A.R., Ibrahim A., 2011. Effects of olive oil on lipid profiles and blood glucose in type 2 diabetic patients. Int. J. Diabetes. Metab.; 19:19-22.

Al-Khazraji, S.M., Al-Shamaony, L.A., Twaij, H.A., 1993. Hypoglycaemic effect of Artemisia herba-alba Asso. I. Effect of different parts and influence of the solvent on hypoglycaemic activity. Journal of Ethnopharmacology 40, 163-166.

Al-Shamouny, L., Al-Khazraji, S.M., Twaij, H.A.A., 1994. Hypoglycaemic effect of Artemisia herba alba. II. Effect of a valuable extract on some blood parameters in diabetic animals. Journal of Ethnopharmacology 43, 167-171. 


\section{Orch et al. J. Appl. Biosci. Étude ethnobotanique des plantes médicinales utilisées dans le}

traitement du diabète, et des maladies cardiaques dans la région d'Izarène (Nord du Maroc)

Andrianova, I.V., Fomchenkov, I.V., Orekhov, A.N., 2002. Hypotensive effect of long-acting garlic tablets allicor (a double-blind placebocontrolled trial). Terapevticheskii Arkhiv 74, 76-78.

Anyinam, C - 1995 - Ecology and ethnomedicine: exploring links between current environmental crisis and indigenous medical practices. Social Science and Medicine 4: 321-329.

Aquel, M., Hadidi, M., 1991. Direct relaxant effect of Peganum harmala seed extract on smooth muscles of rabbit and guinea pig. International Journal of Pharmacognosy 29, 176-182.

Askarn O., 1982. Comportement des Résineux dans l'Arboretum d'Izarène. Mémoire de Troisième Cycle Agronomie, Institut Agronomique et Vétérinaire Hassan II, Rabat, Maroc, $122 \mathrm{p}$.

Beaux, D., Fleurentin, J., Mortier, F., 1997. Diuretic action of hydroalcohol extracts of Foeniculum vulgare var. dulce (D.C.) roots in rats. Phytotherapy Research 11, 320-322.

Bellakhdar, J. 1997 - La pharmacopée marocaine traditionnelle. Médecine arabe ancienne et savoirs populaires. Editions Le Fennec, Casablancal lbis Press. 764 p. Paris.

Benkhnigue O., Zidane L., Fadli M., Elyacoubi H., Rochdi A. \& Douira A., 2010 ethnobotanique des plantes médicinales dans la région de Mechraa Bel Ksiri (Région du Gharb du Maroc). Barc. 53 : 191-216.

Bennani-Kabchi, N., Fdhil, H., Cherrah, Y., El Bouayadi, F., Kehel, L., Marquie, G., 2000. Therapeutic effect of Olea europaea var. oleaster leaves on lipidic and carbohydrate metabolism in obese and prediabetic sand rats (Psammomys obesus). Annales Pharmaceutiques Francaises 58, 271-277.

Bnouham M., Mekhfi H., Legssyer A., Ziyyat A., 2002. Medicinal plants used in the treatment of diabetes in Morocco. Int. J. Diabetes Metab.; 10: $33-50$.

Bnouham, M., Merhfour, F.Z., Ziyyat, A., Mekhfi, H., Aziz, M., Legssyer, A., 2003. Antihyperglycemic activity of the aqueous extract of Urtica dioica. Fitoterapia 74, 677681.

Borgniet L., Long M., Capel A-C., Bouillon C., Morge D., Ganteaume A., Lampin C., Jappiot M., Curt T., Machrouh A., Sesbou A., Mantzavelas A., Apostolopoulou I., Partozis T., Gitas I., Marell
A., Cassagne N., Pimont F., Rigolot E., Morsdorf F., Koetz B. and Allgower B. 2009. Development of an easy to use tool to recognize and map fuel models. Deliverable D5.1-6 of the Integrated Project "Fire Paradox", Project no. FP6-018505. European Commission. $112 p$.

Campos, K.E., Diniz, Y.S., Cataneo, A.C., Faine, L.A., Alves, M.J., Novelli, E.L., 2003. Hypoglycaemic and antioxidant effects of onion, Allium cepa: dietary onion addition, antioxidant activity and hypoglycaemic effects on diabetic rats. International Journal of Food Sciences \& Nutrition 54, 241-246.

Circosta, C., Occhiuto, F., Toigo, S., Gregorio, A., 1986. Studio comparative dell'attività cardiovascolare di germogli e di foglie di Olea europaea. I. attività elettrica e sulla pressione arteriosa. Pharmacia mediterranea 16, 157.

Devi, B.A., Kamalakkannan, N., Prince, P.S., 2003. Supplementation of fenugreek leaves to diabetic rats. Effect on carbohydrate metabolic enzymes in diabetic liver and kidney. Phytotherapy Research 17, 1231-1233.

Eddouks, M., Maghrani, M., Lemhadri, A., Ouahidi, M.L., Jouad, H., 2002. Ethnopharmacological survey of medicinal plants used for the treatment of diabetes mellitus, hypertension and cardiac diseases in the south-east region of Morocco (Tafilalet). Journal of Ethnopharmacology 82, 97-103.

Eddouks, M., Lemhadri, A., Michel, J.-B., 2004. Caraway and caper: potential antihyperglycaemic plants in diabetic rats. Journal of Ethnopharmacology 94, 143-148.

Eddouks M., Ouahidi M.L., Farid O., Moufid A., Khalidi A., Lemhadri A., 2007. L'utilisation des plantes médicinales dans le traitement du diabète au Maroc. Phytothérapie ; 5: 194-203.

Eidi, M., Eidi, A., Zamanizadeh, M., 2005. Effect of Salvia officinalis $L$. leaves on serum glucose and insulin in healthy and streptozotocininduced diabetic rats. Journal of Ethnopharmacology 100, 310-313.

Eidi A., Eidi M., Sokhteh M., 2007. Effect of fenugreek (Trigonella foenum-graecum L.) seeds on serum parameters in normal and streptozotocin-induced diabetic rats. Nutr. Res.; 27:728-733. 


\section{Orch et al. J. Appl. Biosci. Étude ethnobotanique des plantes médicinales utilisées dans le}

traitement du diabète, et des maladies cardiaques dans la région d'Izarène (Nord du Maroc)

El Bardai, S., Lyoussi, B.,Wibo, M., Morel, N., 2001. Pharmacological evidence of hypotensive activity of Marrubium vulgare and Foeniculum vulgare in spontaneously hypertensive rat. Clinical and Experimental Hypertension 23, 329-343.

El-Dakhakhny, M., Mady, N., Lembert, N., Ammon, H.P.T., 2002. The hypoglycemic effect of Nigella sativa oil is mediated by extrapancreatic actions. Planta Medica 68, 465-466.

El-Demerdash, F.M., Yousef, M.I., El-Naga, N.I., 2005. Biochemical study on the hypoglycemic effects of onion and garlic in alloxan-induced diabetic rats. Food \& Chemical Toxicology 43, 57-63.

Elfellah, M.S., Akhtar, M.H., Khan, M.T., 1984. Antihyperglycaemic effect of an extract of Myrtus communis in streptozotocin-induced diabetes in mice. Journal of Ethnopharmacology 11, 275-281.

El-Hilaly, J., Lyoussi, B., 2002. Hypoglycaemic effect of the lyophilised aqueous extract of Ajuga iva in normal and streptozotocin induced diabetic rats. Journal of Ethnopharmacology 80, 109113.

El-Hilaly, J., Lyoussi, B.,Wibo, M., Morel, N., 2004. Vasorelaxant effect of the aqueous extract of Ajuga iva in rat aorta. Journal of Ethnopharmacology 93, 69-74.

El Tahir, K.E.H., Ashour, M.M.S., Al-Harbi, M.M., 1993. The cardiovascular actions of the volatile oil of the black seed (Nigella sativa) in rats: elucidation of the mechanism of action. General Pharmacology 24, 1123-1131.

Erenmemisoglu, A., Saraymen, R., Ustun, S., 1997. Effect of Rosmarinus officinalis leave extract on plasma glucose levels in normoglycaemic and diabetic mice. Pharmazie 52, 645-646.

Fararh, K.M., Atoji, Y., Shimizu, Y., Shiina, T., Nikami, H., Takewaki, T., 2004. Mechanisms of the hypoglycaemic and immunopotentiating effects of Nigella sativa L. oil in streptozotocininduced diabetic hamsters. Research in Veterinary Science 77, 123-129.

Farzami, B., Ahmadvand, D., Vardasbi, S., Majin, F.J., Khaghani, Sh., 2003. Induction of insulin secretion by a component of Urtica dioica leave extract in perfused Islets of Langerhans and its in vivo effects in normal and streptozotocin diabetic rats. Journal of Ethnopharmacology 89, 47-53.

Fennane, M., Ibn Tatt ou, M., Mathez, J., Ouyahya, A. \& El Oualidi, J. 1999 - Flore pratique du Maroc, Vol. I. Trav. Inst. Sci. Rabat, série botanique 36.558 p. Rabat.

Fennane, M. \& Ibn Tatt ou, M. 2005 - Flore vasculaire du Maroc Inventaire et Chorologie, Tome I. Trav. Inst. Sci. Rabat, série botanique 37. 483 p. Rabat.

Fitzpatrick, D.F., Hirschfield, S.L., Coffey, R.G., 1993. Endothelium dependent vasorelaxing activity of wino other grape products. American Journal of Physiology 265, 774-778.

Fluck H. Petit Guide Panoramique des Herbes Medicinales. Delachaux et Nestlé Publishers, Lausanne, Switzerland, 1973, p. 119.

Gallagher, A.M., Flatt, P.R., Duffy, G., Abdel-Wahab, Y.H.A., 2003. The effects of traditional antidiabetic plants on in vitro glucose diffusion. Nutrition Research 23, 413-424.

Ghourri M., Zidane L., Douira A., 2013. Usage des plantes médicinales dans le traitement $\mathrm{du}$ Diabète Au Sahara marocain (Tan -Tan). Journal of Animal \&Plant Sciences. Vol.17, Issue 1: 2388-2411.

Godron, M. 1971 - Essai sur une approche probabiliste de l'écologie des végétaux. Thèse Univ. Sci. Tech. Languedoc. 247 p. Montpellier.

Godron, M. 1982 - Analyse fréquentielle de l'écologie des espèces dans les communautés. Edit. Masson. Paris, New York, Barcelone, Milon, Mexico, Rio de Janeiro. 163p.

Gupta, A., Gupta, R., Lal, B., 2001. Effect of Trigonella foenum-graecum (fenugreek) seeds on glycaemic control and insulin resistance in type 2 diabetes mellitus: a double blind placebo controlled study. Journal of the Association of Physicians of India 49, 10571061.

Haloui, M., Louedec, L., Michel, J.P., Lyoussi, L., 2000. Experimental diuretic effects of Rosmarinus officinalis and Centaurium erythraea. Journal of Ethnopharmacology 71, 465-472.

Hamza, N., Berke, B., Cheze, C., Agli, A.-N., Robinson, P., Gin, H., Moore, N., 2010. Prevention of type 2 diabetes induced by high fat diet in the C57BL/6J mouse by two medicinal plants used in traditional treatment of diabetes in the east 
of Algeria. Journal of Ethnopharmacology 128, 513-518.

Hawsawi, Z.A., Ali, B.A., Bamosa, A.O., 2001. Effect of Nigella sativa (black seed) and thymoquinone on blood glucose in albino rats. Annals of Saudi Medicine 21, 242-244.

Herrera-Arellano, A., Aguilar-Santamaría, L., GarcíaHernández, B., Nicasio- Torres, P., Tortoriello, J., 2004. Clinical trial of Cecropia obtusifolia and Marrubium vulgare leaf extracts on blood glucose and serum lipids in type 2 diabetics. Phytomedicine 11, 561-566.

Hmamouchi, M. 2001 - Les plantes médicinales et aromatiques marocaines. 2éme. Ed. $389 p$.

Jaouhari J.T., Lazrek H.B., Seddik A., Jana M., 1999. Hypoglycaemic response to Zygophyllum gaetulum extracts in patients with non-insulindependent diabetes mellitus. J. Ethnopharmacol.; 64: 211-217.

Jaouhari, J.T., Lazrek, H.B., Jana, M., 2000. The hypoglycemic activity of Zygophyllum gaetulum extracts in alloxan-induced hyperglycemic rats. Journal of Ethnopharmacology 69, 17-20.

Jayakumar G, Ajithabai M D, Sreedevi S, Viswanathan P K, Remeshkumar. 2010. Ethnobotanical survey of the plangts used in the treatment of diabetes. Indian Journal of Traditional Knowledge, 9(1);100-104.

Jelodar, G.A., Maleki, M., Motadayen, M.H., Sirus, S., 2005. Effect of fenugreek, onion and garlic on blood glucose and histopathology of pancreas of alloxan-induced diabetic rats. Indian Journal of Medical Sciences 59, 64-69.

Jeruto Pascaline, Lukhoba Catherine, Ouma George, Otieno Dennis, Mutai Charles, 2008. -An ethnobotanical study of medicinal plants used by the Nandi people in Kenya. Journal of Ethnopharmacology 116 (2008) 370-376.

Jouad, H., Haloui, M., Rhiouani, H., El-Hilaly, J., Eddouks, M., 2001. Ethnobotanical survey of medicinal plants used for the treatment of diabetes, cardiac and renal diseases in the North centre region of Morocco (FezBoulemane). Journal of Ethnopharmacology 77, 175-182.

Jouad H., Maghrani M., Eddouks M., 2002. Hypoglycemic effect of aqueous extract of Ammi visnaga in normal and streptozotocin- induced diabetic rats. J. Herb. Phamacother.; 2: 19-29.

Jouad, H., Maghrani, M., El Hassani, R.A., Eddouks, M., 2003. Hypoglycemic activity of aqueous extract of Eucalyptus globulus in normal and streptozotocin-induced diabetic rats. Journal of Herbs, Spices \& Medicinal Plants 10, 19-28.

Kahouadji, M.S., 1995 - Contribution a une étude ethnobotanique des plantes médicinales dans le Maroc orientale - Mem. Doc. 3eme cycl. (ined.). Fac. Sci., Univ. Mohamed I, Oujda, Maroc. 206 pp.

Kanter, M., Meral, I., Yener, Z., Ozbek, H., Demir, H., 2003. Partial regeneration/ proliferation of the beta-cells in the Islets of Langerhans by Nigella sativa L. in streptozotocin-induced diabetic rats. Tohoku Journal of Experimental Medicine 201, 213-219.

Kelkar S.M., Kaklij G.S. Bapat V.A., 2001. Determination of hypoglycemic activity in Allium cepa (Oignon) tissue cultures. Indian J. Biochem. Biophys.; 38: 277-279.

Kumari, K., Augusti, K.T., 2002. Antidiabetic and antioxidant effects of Smethyl cysteine sulfoxide isolated from onions (Allium cepa L.) as compared to standard drugs in alloxan diabetic rats. Indian Journal of Experimental Biology 40, 1005-1009.

Labhal, A., Settaf, A., Cherrah, Y., Ettaib, A., El Kabbaj, S., Amrani, A., El Fassi, R., Hassar, M., Seqat, M., Slaoui, A., 1994. Action antihypertensive de Nigella sativa chez le rat spontanément hypertendu (SHR). Actes du IV Congrès National d'Endocrinologie Comparée, Marrakech 15-17Décembre, p. 106 (abstracts).

Labhal, A., Settaf, A., Zalagh, F., Cherrah, Y., Hassar, M., Slaoui, A., 1999. Propriétés antidiabétiques des graines de Nigella sativa chez le Merione Shawi obèse et diabétique. Esp'erance m'edicale 47, 72-74.

Le, P.M., Benhaddou-Andaloussi, A., Elimadi, A., Settaf, A., Cherrah, Y., Haddad, P.S., 2004. The petroleum ether extract of Nigella sativa exerts lipid lowering and insulin-sensitizing actions in the rat. Journal of Ethnopharmacology 94, 251-259.

Lefèbvre P., 2008. -La pandémie de diabète : un fléau cardiovasculaire et une menace pour les systèmes de sante et l'économie mondiale. 
Médecine des maladies métaboliques; 2:16979.

Maghrani, M., Zeggwagh, N.A., Michel, J.-P., Eddouks, M., 2005. Antihypertensive effect of Lepidium sativum $L$. in spontaneously hypertensive rats. Journal of Ethnopharmacology 100, 193-197.

Malik, Z.A., Siddiqui, S., 1981. Hypotensive effect of freeze-dried garlic (Allium sativum) sap in dogs. Journal of Pakistan Medical Association $13,12-13$.

Mansi, K.M.S., 2006. Effects of oral administration of water extract of Nigella sativa on the hypothalamus pituitary adrenal axis in experimental diabetes. International Journal of Pharmacology 2, 104-109.

Mansi, K., Amneh, M., Nasr, H., 2007. The hypolipidemic effects of Artemisia sieberi (A. herba-alba) in alloxan induced diabetic rats. International Journal of Pharmacology 3, 487491.

Marrif, H.I., Ali, B.H., Hassan, K.M., 1995. Some pharmacological studies on Artemisia herbaalba Asso. in rabbits and mice. Journal of Ethnopharmacology49, 51-55.

Mathew, P.T., Augusti, K.T., 1975. Hypoglycaemic effects of onion, Allium cepa Linn. on diabetes mellitus-a preliminary report. Indian Journal of Physiology \& Pharmacology 19, 213-217.

Mehdioui R. et Kahouadji A: 2007. Étude ethnobotanique auprès de la population riveraine de la forêt d'Amsittène: cas de la Commune d'Imi n'Tlit (Province d'Essaouira). Bulletin de l'Institut Scientifique, Rabat, section Sciences de la Vie n²9: 11-20.

M.S, Ministère de la Santé, 2007. - Santé, vision 2020. $49 p$.

Miura, T., Toyoda, H., Miyake, M., Ishihara, E., Usami, M., Tanigawa, K., 1996. Hypoglycemic action of stigma of Zea mays $L$. in normal and diabetic mice. Natural Medicines 50, 363-365.

Negre, R. 1961 \& 1962 - Petite flore des régions arides du Maroc occidental. C.N.R.S. 979 p. Paris.

Novaes, A.P., Rossi, C., Poffo, C., Pretti Junior, E., Oliveira, A.E., Schlemper, V., Niero, R., Cechinel-Filho, V., Burger, C., 2001. Preliminary evaluation of the hypoglycemic effect of some Brazilian medicinal plants. Therapie 56, 427-430.

OMS, Organisation mondiale de la Santé, 2012. Statistiques sanitaires mondiales. $175 \mathrm{p}$.
Onal, S., Timur, S., Okutucu, B., Zihnioglu, F., 2005. Inhibition of alphaglucosidase by aqueous extracts of some potent antidiabetic medicinal herbs. Preparative Biochemistry \& Biotechnology 35, 29-36.

Ozougwu J.C., 2011. Anti-diabetic effects of Allium cepa (onions) aqueous extracts on alloxan induced diabetic Rattus novergicus. J. Med. Plants Res. ; 5(7): 1134-1139.

Pantoja, C.V., Chiang, L.C., Norris, B.C., Concha, J.B., 1991. Diuretic, natriuretic and hypotensive effects produced by Allium sativum (garlic) in anaesthetized dogs. Journal of Ethnopharmacology 31, 321-331.

Pantoja, C.V., Norris, B.C., Contreras, C.M., 1996. Diuretic, natriuretic effects of chromatographically purified fraction of garlic (Allium sativum). Journal of Ethnopharmacology 52, 101_/105.

Prance, G.T., 1991. What is ethnobotany today? Journal of Ethnopharmacology 32, 209-216.

Quézel, P. \& Santa, S. 1962 \& 1963 - Nouvelle flore d'Algérie et des régions désertiques méridionales. C.N.R.S. $1170 \mathrm{p}$.

Raju, J., Gupta, D., Rao, A.R., Yadava, P.K., Baquer, N.Z., 2001. Trigonella foenum graecum (fenugreek) seed powder improves glucose homeostasis in alloxan diabetic rat tissues by reversing the altered glycolytic, gluconeogenic and lipogenic enzymes. Molecular \& Cellular Biochemistry 224, 45-51.

RBPM, Recommandations de Bonnes Pratiques Médicales. -L'hypertension artérielle de l'adulte. Affection Longue Durée. ALD 14/ Réf : 0.3.88.01. 83p.

Rchid, H., Chevassus, H., Nmila, R., Guiral, C., Petit, P., Chokairi, M., Sauvaire, Y., 2004. Nigella sativa seed extracts enhance glucose-induced insulin release from rat-isolated Langerhans islets. Fundamental \& Clinical Pharmacology 18, 525-529.

Riyad, M.A., Abdul-Salam, S.A., Mohammad, S.S., 1988. Effect of fenugreek and lupine seeds on the development of experimental diabetes in rats. Planta Medica 54, 286-290.

Roman Ramos, R., Alarcon-Aguilar, F., Lara-Lemus, A., Flores-Saenz, J.L., 1992. Hypoglycemic effect of plants used in Mexico as antidiabetics. Archives of Medical Research $23,59-64$. 
Roman-Ramos, R., Flores-Saenz, J.L., Alarcon-Aguilar, F.J., 1995. Antihyperglycemic effect of some edible plants. Journal of Ethnopharmacology 48, 25-32.

Salhi S., Fadli M., Zidane L., Douira A., 2010. Études floristique et ethnobotaniques des plantes médicinales de la ville de Kénitra (Maroc). Lazaroa 31 : 133-146.

Sepici, A., Gurbuz, I., Cevik, C., Yesilada, E., 2004. Hypoglycaemic effects of myrtle oil in normal and alloxan-diabetic rabbits. Journal of Ethnopharmacology 93, 311-318.

Sharma, R.D., Raghuram, T.C., Rao, N.S., 1990. Effect of fenugreek seeds on blood glucose and serum lipids in type I diabetes. European Journal of Clinical Nutrition 44, 301-306.

Sheela, C.G., Augusti, K.T., 1992. Antidiabetic effects of S-allyl cysteine sulphoxide isolated from garlic Allium sativum Linn. Indian Journal of Experimental Biology 30, 523-526.

Sial, A.Y., Ahmad, S.I., 1982. Study of the hypotensive action of garlic extract in experimental animals. Journal of the Pakistan Medical Association $32,237-239$.

Sijelmassi, A. 1993 - Les plantes médicinales du Maroc, 6ème édition. Fennec. 285 p. Casablanca.

Skim, F., Lazrek, H.B., Kaaya, A., El Amri, H., Jana, M., 1999. Pharmacological studies of two antidiabetic plants: Globularia alypum and Zygophyllum gaetulum. Therapie 54, 711-715.

Somova, L.I., Shode, F.O., Ramnanan, P., Nadar, A., 2003. Antihypertensive, antiatherosclerotic and antioxidant activity of triterpenoids isolated from Olea europaea, subspecies africana leaves. Journal of Ethnopharmacology 84, 299-305.

SPEF, 2004. - Incendie du massif forestier d'Izarene du 23 au 25 aout 2004. Service Provincial des Eaux et Forêts de Chefchaouen ; 17p.

Swanston-Flatt, S.K., Day, C., Flatt, P.R., Gould, B.J., Bailey, C.J., 1989. Glycaemic effects of traditional European plant treatments for diabetes. Studies in normal and streptozotocin diabetic mice. Diabetes Research 10,69-73.

Swanston-Flatt, S.K., Day, C., Bailey, C.J., Flatt, P.R., 1990. Traditional plant treatments for diabetes.
Studies in normal and streptozotocin diabetic mice. Diabetologia 33, 462-464.

Tahraoui, A., El-Hilaly, J., Israili, Z.H., Lyoussi, B., 2007. Ethnopharmacological survey of plants used in the traditional treatment of hypertension and diabetes in south-eastern Morocco (Errachidia province). Journal of Ethnopharmacology 110, 105-117.

Tahri Nabila, El basti Abdelkrim, Zidane Lahcen, Rochdi Atmane, Douira Allal, 2012. Kastamonu Üni., Orman Fakültesi Dergisi (Kastamonu Univ., Journal of Forestry Faculty), 12 (2): 192-208.

Tastekin, D., Atasever, M., Adiguzel, G., Keles, M., Tastekina, A., 2006. Hypoglycaemic effect of Artemisia herba alba in experimental hyperglycaemic rats. Bulletin of the Veterinary Institute in Pulawy 50, 235-238.

Trabi Fézan H., Irié Guy M., N'gaman Kohué C.C. \& Mohou Clejesson H.B., 2008. - Études de quelques plantes thérapeutiques utilisées dans le traitement de l'hypertension artérielle et du diabète : deux maladies émergentes en Côte d'Ivoire. Sciences \& Nature Vol. $5 \mathrm{~N}^{\circ} 1$ : $39-48$.

Twaij H.A., Al-Badr A.A., 1988. Hypoglycemic activity of Artemisia herba-alba. J. Ethnopharmacol., 24: 123-126.

Valdes, B., Rejdali, M., Achhal el Kadmiri, A., Jury, J.L., \& Monts err at, J.M. 2002 - Catalogues des plantes vasculaires du Nord du Maroc, incluant des clés d'identification, 2 Volumes. 1498 p. Madrid.

Weniger, B., 1991. Interest and limitation of a global ethnopharmacological survey. Journal of Ethnopharmacology 32, 37-41.

Zaoui, A., Cherrah,Y., Lacaille-Dubois, M.A., Settaf, A., Amarouch, H., Hassar, M., 2000. Diuretic and hypotensive effects of Nigella sativa on the spontaneously hypertensive rat. Therapie 55 , 379-382.

Ziyyat, A., Legssyer, A., Mekhfi, H., Dassouli, A., Serhrouchni, M., Benjelloun, W., 1997. Phytotherapy of hypertension and diabetes in oriental Morocco. Journal of Ethnopharmacology 58, 45_/54. 